\title{
Wavelength shifts of cladding-mode resonance in corrugated long-period fiber gratings under torsion
}

\author{
Oleg V. Ivanov and Lon A. Wang
}

\begin{abstract}
A finite deformation theory of elasticity and a theory of nonlinear photoelasticity are applied to describe the wavelength shifts of cladding-mode resonance in corrugated long-period fiber gratings under torsion. The deformation of fiber is found by use of the Murnaghan model of a solid elastic body. The quadratic photoelastic effect that is proportional to the second-order displacement gradient is investigated and compared with the classical photoelastic effect. The electromagnetic field in the twisted corrugated structure is presented as a superposition of circularly polarized modes of the etched fiber section. The wavelength shift is found to be proportional to the square of the twist angle. As predicted by our theory, a wavelength shift of the same nature has been found in a conventionally photoinduced long-period fiber grating. (C) 2003 Optical Society of America

OCIS codes: $\quad 050.2770,060.2370,060.2340$.
\end{abstract}

\section{Introduction}

Long-period fiber gratings (LPFGs) provide coupling between the core mode and the copropagating cladding modes of single-mode optical fibers and can be used as band-rejection filters, ${ }^{1}$ optical sensors, ${ }^{2,3}$ and gain flatteners. ${ }^{4,5}$ The advantages of these devices are low insertion loss and low backreflection. In comparison with fiber Bragg gratings, LPFGs are much more sensitive to the variation of fiber properties and surrounding medium parameters because of the phase-matching condition between copropagating modes and propagation of light in fiber cladding. Any modulation in the guiding property of cladding modifies the spectral response of a LPFG, and this phenomenon can be utilized to tune the spectrum of a LPFG. However, the overall sensitivity of a LPFG can be low because a change in the cladding properties is often compensated by the accompanying change in the core as well. For example, in the case of a strained LPFG, when both the core and the cladding are equally strained, the decrease in refractive index of the cladding is compensated by nearly the

O. V. Ivanov and L. A. Wang (lon@ccms.ntu.edu.tw) are with the Department of Electrical Engineering and Institute of ElectroOptical Engineering, National Taiwan University, Taipei 10617, Taiwan.

Received 13 August 2002; revised manuscript received 15 January 2003.

0003-6935/03/132264-09\$15.00/0

(C) 2003 Optical Society of America same amount of the core. ${ }^{6}$ To increase the sensitivity of a LPFG, therefore, the core and the cladding must undergo different modifications.

The following methods can be employed to achieve separate influence in the core and the cladding: bend a fiber, ${ }^{7,8}$ change the ambient refractive index, ${ }^{2,9}$ cladding etching, ${ }^{10}$ and a novel method of periodically cladding etching to create a corrugated LPFG (CLPFG). ${ }^{3,11-13}$ The periodic etching of fiber cladding provides a unique opportunity to control the parameters of a LPFG. By imposing the corrugated structure upon a fiber Bragg grating, one can form a superstructure grating with tunable reflectance. ${ }^{14}$ It has been shown that a CLPFG is highly sensitive to applied tensile strain, torsion, and bending. ${ }^{3}$ The resonance loss varies and the resonant wavelength remains stable under a tensile strain; however, the resonant wavelength shifts with the applied torsion. Both resonant loss and wavelength vary in a bent CLPFG. The transfer matrix method was used to describe the behavior of the CLPFG under tensile strain. ${ }^{6}$ The transmission spectra of a strained CLPFG have been calculated and compared with the experimental results. The wavelength shifts (WSs) of resonant peaks of a CLPFG under torsion was explained to be due to the change of propagation constants of the cladding modes. However, the nature and the mechanism of such a change are yet to be explored.

Based on the finite deformation theory of elasticity, the theory of nonlinear photoelasticity, and the coupled-mode method, we explain spectral responses 


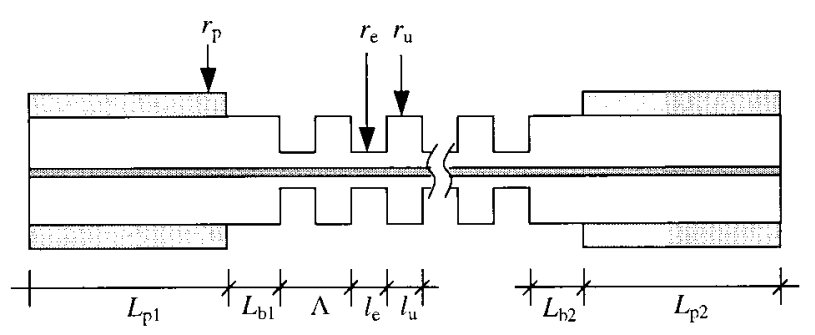

Fig. 1. Schematic structure of a CLPFG.

of a CLPFG under torsion. First, we analyze the deformation of the fiber with a CLPFG and show that the finite deformation theory of elasticity shall be applied. We found the deformation of a twisted fiber by using the Murnaghan model of a solid elastic body. Second, we obtained the quadratic tensor of photoelasticity that is described by an independent displacement gradient variable and show its difference from the same tensor described by use of the strain variable. Third, we employed the method of coupled circularly polarized modes to describe the light propagation in a twisted CLPFG. The circular modes of the etched section of twisted fiber are regarded as unperturbed. The nonlinear torsion-induced variation of a dielectric tensor of fiber and unetched cladding introduce a perturbation that contributes to the propagation constants of the core and cladding circular modes through their self-coupling. Finally, we considered the phase-matching condition, derived the WSs of resonant peaks under torsion, and made comparisons with experimental results.

\section{Finite Deformation of Fiber under Strong Torsion}

The schematic structure of a CLPFG is shown in Fig. 1. It consists of pristine fiber $\left(L_{p}=L_{p 1}+L_{p 2}\right)$, bare fiber without a coating $\left(L_{b}=L_{b 1}+L_{b 2}\right)$, and etched $\left(l_{e}\right)$ and unetched sections of fiber $\left(l_{u}\right)$ with radii $r_{p}, r_{e}$, and $r_{u}$, respectively. When the fiber is twisted at its ends, the torsion is inhomogeneously distributed over different fiber sections. This distribution can be found from equations of torques created in each section. Solving this problem, we can find the twist per unit length of the etched section of a CLPFG:

$$
\tau_{e}=\phi\left[l_{e}+\left(\frac{r_{e}}{r_{u}}\right)^{4}\left(l_{u}+L_{b}\right)+\frac{1}{k}\left(\frac{r_{e}}{r_{u}}\right)^{4} L_{p}\right]^{-1},
$$

where $\phi$ is the twist angle, $\tau$ is the twist per unit length, and $k$ is the parameter that defines the relation between torque of coated and bare fibers.

The torque created by a cylinder is proportional to the fourth power of its radius, ${ }^{15}$ therefore, the relation between the specific twists of etched and unetched sections of fiber is given by

$$
\tau_{e} / \tau_{u}=\left(r_{u} / r_{e}\right)^{4} .
$$

In all the experiments with CLPFGs, the fiber radius in the etched section was at least two times smaller than the radius of the unetched section. It then follows that the twist of the unetched section is at least 16 times smaller than the twist of the etched section and can be neglected. Another result of this relation is that, in spite of the short length of the etched section relative to the whole fiber length, a large part of the twist is concentrated at the etched sections of fiber.

The strain tensor to describe deformation of a medium is defined as

$$
\widehat{\eta}=\frac{1}{2}\left(\widehat{J}^{T} \widehat{J}-\widehat{I}\right),
$$

where $J_{i j}=\partial x_{i}{ }^{\prime} / \partial x_{j}$ is the Jacobian matrix of transformation from the initial state of medium $\left(x_{j}\right)$ to the final strained state $\left(x_{i}{ }^{\prime}\right)$, and $\bar{I}$ is the unit matrix. Hereafter a hat is used to denote a matrix or a tensor. From the definition, it follows that the strain tensor is symmetric. Any symmetric tensor has three invariants that are insensitive to rotation of the reference frame. To describe nonlinear elasticity, the energy as a function of these invariants is employed. One of the most famous functions is introduced by Murnaghan. ${ }^{16}$ His energy function contains nonlinear terms of the third order of the strain tensor and five parameters that define elastic properties of a medium: $\mu, \lambda$ are Lame linear elastic constants; $l, m, n$ are nonlinear Murnaghan parameters.

Let us consider the torsion of a fiber cylinder with radius $R$. We assume that the deformed cylinder is in equilibrium under the applied torque to its ends. By employing the linear theory, a pure shear strain is obtained; however, as will be shown in Section 4, such a strain cannot produce the WS. Therefore, by adopting a second-order approximation, one can describe the deformation of the cylinder by the transformation from the initial reference frame $(r, \theta, z)$ to the final one $\left(r^{\prime}, \theta^{\prime}, z^{\prime}\right)^{16}$ :

$$
\begin{aligned}
r^{\prime} & =r+\tau^{2} \nu, \quad \theta^{\prime}=\theta+\tau z, \quad z^{\prime}=z+\tau^{2} s, \\
\nu & =C R^{2} r+F r^{3}, \quad s=D R^{2} z, \\
& =\frac{2 \mu-\lambda-m+\frac{3}{4} n}{8(\lambda+2 \mu)},
\end{aligned}
$$

where $C$ and $D$ are functions of linear $\mu, \lambda$ and nonlinear $l, m, n$ parameters of elasticity and can be found from the solution of the following equations:

$$
\begin{aligned}
2(\lambda+\mu) C & +\lambda D+\frac{1}{16(\lambda+2 \mu)} \\
& \times\left[20 \lambda \mu+24 \mu^{2}+4 \mu m+(2 \lambda+\mu) n\right]=0 \\
2 \lambda C+(\lambda & +2 \mu) D+\frac{1}{16(\lambda+2 \mu)} \\
& \times\left(24 \lambda \mu+16 \mu^{2}+8 \mu m+3 \lambda n\right)=0 .
\end{aligned}
$$

From Eqs. (4) we can find the displacement gradient $u_{i j}=\partial\left(x_{i}{ }^{\prime}-x_{i}\right) / \partial x_{j}$ and the strain tensor of the 
twisted cylinder, taking into account the first- and the second-order terms in $\tau$ :

$$
\begin{aligned}
& u_{r r}=\eta_{r r}=\tau^{2} \mathrm{~d} \nu / \mathrm{d} r, \quad u_{\theta \theta}=\eta_{\theta \theta}=\tau^{2} \nu / r, \\
& u_{z z}=\tau^{2} \mathrm{~d} s / \mathrm{d} z, \quad \eta_{z z}=\tau^{2}\left(\mathrm{~d} s / \mathrm{d} z+r^{2} / 2\right), \\
& u_{\theta z}=\tau r, \quad \eta_{\theta z}=\eta_{z \theta}=\tau r / 2,
\end{aligned}
$$

with other components being zero. In addition to the shear strain along the hoop direction, the cylinder is therefore longitudinally stretched if $D>0$ or compressed if $D<0$. There is also an inhomogeneous deformation in the radial direction, which depends on the radius and is defined by the constants $F$ and $C$.

\section{Linear and Quadratic Photoelasticity}

When a body is in a strained state there exists a change in the dielectric tensor $\varepsilon_{i j}$ or its reciprocal, the impermeability tensor $\xi_{i j}$. Both tensors are symmetric for nonmagnetic media. The classical interpretation of the photoelastic effect is based on the phenomenological relationship between the impermeability tensor and the strain tensor according to

$$
\xi_{i j}-\xi_{i j}^{(0)}=p_{i j k l} \eta_{k l},
$$

where $p_{i j k l}$ is the photoelastic tensor, ${ }^{17}$ and $\xi_{i j}{ }^{(0)}$ is the impermeability tensor in the unstrained state. Because $\xi_{i j}$ and $\eta_{k l}$ are both symmetric tensors, $p_{i j k l}$ is symmetric after permutation of indices $i$ and $j$ and after permutation of $k$ and $l$. An analogous relationship can be written for the dielectric tensor:

$$
\varepsilon_{i j}-\varepsilon_{i j}{ }^{(0)}=-\varepsilon_{i m}{ }^{(0)} \varepsilon_{n j}{ }^{(0)} p_{m n k l} \eta_{k l},
$$

which is equivalent to Eq. (7) when the strain is infinitesimal.

Since the deformation of fiber under torsion cannot be considered as infinitesimal, classical interpretation becomes inadequate. Nelson and Lax showed ${ }^{18}$ that not strain nor stress tensors but the displacement gradient is the proper variable that defines the optical properties of a medium when finite deformations of an elastic medium are involved. With the account of second-order terms of the displacement gradient, expansion of the dielectric tensor can be written as

$\varepsilon_{i j}=\varepsilon_{i j}{ }^{(0)}+\varepsilon_{i j}{ }^{(1)}+\varepsilon_{i j}{ }^{(2)}=\varepsilon_{i j}{ }^{(0)}+\alpha_{i j k l} u_{k l}+\gamma_{i j k l m n} u_{k l} u_{m n}$,

where $\alpha_{i j k l}$ and $\gamma_{i j k l m n}$ are the first- and the secondorder tensors for gradient representation of the photoelastic effect, respectively. Tensors $\alpha_{i j k l}$ and $\gamma_{i j k l m n}$ are not symmetric after permutation of indices $k$ and $l$ and $m$ and $n$ because $u_{i j}$ is generally not symmetric. The displacement gradient is considered a correct variable because the rotation of a medium, which is described by an antisymmetric combination of a displacement gradient, is eliminated in the symmetric strain tensor. However, the rotation changes the dielectric tensor and should not be neglected.
The classical relationship including the quadratic photoelasticity has the following form ${ }^{19,20 \text { : }}$

$$
\varepsilon_{i j}=\varepsilon_{i j}^{(0)}+q_{i j k l} \eta_{k l}+s_{i j k l m n} \eta_{k l} \eta_{m n},
$$

where $q_{i j k l}$ and $s_{i j k l m n}$ are the first- and the secondorder tensors for representation of the photoelastic effect with a strain variable, respectively. The use of Eq. (10) is justified only when hydrostatic pressure or axial stress is applied to the body and there is no rotation or shear strain of the medium. If we substitute the strain tensor in Eq. (3) into Eq. (10) we obtain

$$
\begin{aligned}
\varepsilon_{i j}= & \varepsilon_{i j}{ }^{(0)}+\frac{1}{2}\left(q_{i j k l}+q_{i j l k}\right) u_{k l}+\frac{1}{4}\left(s_{i j k l m n}+s_{i j l k m n}\right. \\
& \left.+s_{i j k l n m}+s_{i j l k n m}+2 q_{i j k m} \delta_{l n}\right) u_{k l} u_{m n} .
\end{aligned}
$$

where $\delta_{l n}$ is the Kronecker delta function. Three differences are obvious from the comparison with Eq. (9):

(a) the linear term is symmetrized upon indices $k$ and $l$;

(b) the quadratic term is symmetrized upon indices $k$ and $l$ and $m$ and $n$; and

(c) there is an additional term that is a quadratic function of the displacement gradient and is proportional to the linear photoelastic constants.

So, the symmetries of tensors $\widehat{\alpha}, \widehat{\gamma}$ and $\widehat{q}, \widehat{s}$ are different in general. The form of tensor $\alpha$ and the number of nonzero components for different crystallographic systems have been found in Ref. 19, where it is shown that isotropic substances and cubic crystals (nonmagnetic and optically inactive) have no antisymmetric components in $\widehat{\alpha}$. Therefore, the descriptions of linear photoelasticity of an isotropic medium by use of variables of either a strain or a displacement gradient are equivalent to each other.

The situation is different if we take into account quadratic photoelasticity. Tensor $\hat{s}$ of an isotropic medium has 183 nonzero components, four of which are independent parameters. ${ }^{19}$ The tensor $\gamma_{i j k l m n}$ is nonsymmetric after permutation of $k$ and $l$ and $m$ and $l$. From the symmetry of the $\gamma_{i j k l m n}$ it can be shown that the total number of independent second-order photoelastic constants for a crystal of triclinic symmetry is 270. This number decreases with higher symmetry. Since the fiber material is isotropic, we found the second-order photoelastic constants of an isotropic medium in displacement gradient representation. It has also 183 nonzero components, 16 of which are listed below:

$$
\begin{gathered}
\gamma_{x x x x x x}, \gamma_{x x x x y y}, \gamma_{x x x y x y}, \gamma_{x x x y y x}, \gamma_{x x y x y x}, \gamma_{x x y y y y}, \\
\gamma_{x x y y z z}, \gamma_{x x y z y z}, \gamma_{x x y z z y}, \gamma_{x y x x x y}, \gamma_{x y x x y x}, \gamma_{x y z z x y}, \\
\gamma_{x y x z y z}, \gamma_{x y x z z y}, \gamma_{x y z x y z}, \gamma_{x y z x z y},
\end{gathered}
$$

and others can be obtained by symmetry operations with indices of the above components. Seven of 
these components are independent, i.e., there are nine relations among 16 listed components:

$$
\begin{gathered}
\gamma_{x y z x z y}=\gamma_{x x y y z z}-\gamma_{x x x x y y}-\gamma_{x y x z y z} \\
-2 \gamma_{x y x z z y}+\gamma_{x x x x x x} / 2-\gamma_{x x y y y y} / 2, \\
\gamma_{x x x y y x}=\gamma_{x x y y y}-\gamma_{x x y y z z}-\gamma_{x x x y x y} \\
+2 \gamma_{x y x z y z}+2 \gamma_{x y x z z y}, \\
\gamma_{x y z x y z}=\gamma_{x y x z z y}, \quad \gamma_{x x y z y z}=\gamma_{x x x y x y}-2 \gamma_{x y x z y z}, \\
\gamma_{x y x x x y}=\gamma_{x x x x y y} / 2-\gamma_{x x y y z z} / 2+\gamma_{x y x z z y}+\gamma_{x y z x z y}, \\
\gamma_{x x y x y x}=\gamma_{x x y z y z}+2 \gamma_{x y z x z y}, \\
\gamma_{x y x x y x}=\gamma_{x y x x x y}+\gamma_{x y x z y z}-\gamma_{x y z x z y}, \\
\gamma_{x y y z z y}=\gamma_{x x y y y y}-\gamma_{x x y y z z}-\gamma_{x x y z y z}, \\
\gamma_{x y z z x y}=\gamma_{x y x x y x}-\gamma_{x y x z y z}-\gamma_{x y z x y z} .
\end{gathered}
$$

Nonlinear variation of the refractive index of silica with a strain up to $2 \%$ has been measured in experiments with purely hydrostatic pressure. ${ }^{21}$ However, no numerical data on second-order photoelastic constants have been determined.

In experiments with a twisted fiber, the maximum shear strain was approximately $3.5 \%$; therefore, the quadratic effects are approximately three times stronger than those obtained by hydrostatic pressure. Besides, different photoelastic constants are involved in the case of shear strain. The break limit of pristine fiber for a twist-induced shear strain is approximately $12 \%$, and, in principle, strong nonlinear effects are expected.

Since there are no antisymmetric components in $\hat{\alpha}$ for an isotropic medium, this tensor coincides with $\hat{q}$. It has two independent parameters, $p_{11}$ and $p_{12}$, and the following components:

$$
\begin{aligned}
\alpha_{x x x x} & =-\varepsilon^{2} p_{11}, \quad \alpha_{x x y y}=-\varepsilon^{2} p_{12}, \\
\alpha_{x y x y} & =-\varepsilon^{2}\left(p_{11}-p_{12}\right) / 2, \\
p_{11} & =p_{x x x x}, \quad p_{12}=p_{x x y y},
\end{aligned}
$$

where other components can be obtained by symmetry operations with the indices, and $\varepsilon$ is the dielectric permittivity of the isotropic medium without strain.

For the twisted fiber, as we can see from Eq. (6), the shear component of the displacement gradient $u_{\theta z}$ is of the first order of magnitude in the specific twist, whereas the axial components are of the second order. Therefore, we retain only the shear displacement gradient in the quadratic photoelastic effect:

$$
\begin{gathered}
\varepsilon_{i j}{ }^{(2)}=\gamma_{i j y z y z} u_{\theta z} u_{\theta z}, \quad \varepsilon_{r r}{ }^{(2)}=\gamma_{x x y z y z} u_{\theta z}{ }^{2}, \\
\varepsilon_{\theta \theta}{ }^{(2)}=\gamma_{x x x y x y} u_{\theta z}{ }^{2}, \quad \varepsilon_{z z}{ }^{(2)}=\gamma_{x x y x y x} u_{\theta z}{ }^{2},
\end{gathered}
$$

To shorten notations we use $\gamma_{1}, \gamma_{2}, \gamma_{3}$ instead of $\gamma_{x x y z y z}, \gamma_{x x x y x y}, \quad \gamma_{x x y x y x}$, respectively. Substituting
Eqs. (13) and (14) into Eq. (9), we find the dielectric permittivity tensor of a twisted fiber:

$$
\begin{aligned}
& \varepsilon_{r r}=\varepsilon^{(0)}+\varepsilon_{r r}{ }^{(n l)}=\varepsilon^{(0)}+\tau^{2}\left(R^{2} A_{r}+r^{2} B_{r}+r^{2} \gamma_{1}\right), \\
& \varepsilon_{\theta \theta}=\varepsilon^{(0)}+\varepsilon_{\theta \theta}{ }^{(n l)}=\varepsilon^{(0)}+\tau^{2}\left(R^{2} A_{\theta}+r^{2} B_{\theta}+r^{2} \gamma_{2}\right), \\
& \varepsilon_{z z}=\varepsilon^{(0)}+\varepsilon_{z z}{ }^{(n l)}=\varepsilon^{(0)}+\tau^{2}\left(R^{2} A_{z}+r^{2} B_{z}+r^{2} \gamma_{3}\right), \\
& \varepsilon_{\theta z}=\varepsilon_{z \theta}=-\frac{1}{2}\left(p_{11}-p_{12}\right) \tau r \varepsilon^{2},
\end{aligned}
$$

where

$$
\begin{aligned}
& A_{r}=A_{\theta}=-\varepsilon^{2}\left(p_{11} C+p_{12} C+p_{12} D\right), \\
& A_{z}=-\varepsilon^{2}\left(p_{11} D+2 p_{12} C\right), \\
& B_{r}=-\varepsilon^{2}\left(3 p_{11}+p_{12}\right) F, \\
& B_{\theta}=-\varepsilon^{2}\left(p_{11}+3 p_{12}\right) F, \\
& B_{z}=-4 \varepsilon^{2} p_{12} F,
\end{aligned}
$$

and $\widehat{\varepsilon}^{(n l)}$ includes the terms of nonlinear elastic and nonlinear photoelastic effects.

\section{Method of Coupled Circularly Polarized Modes}

The corrugated structure consists of two basic regions: etched and unetched. When the structure is stretched and twisted, the strain is periodically distributed over its length and creates a LPFG. The etched radius is bigger than the mode field diameter of the core mode; therefore, the core mode is not directly affected by corrugations (however, it is affected by inhomogeneous strain induced as a result of corrugation). Because the cladding modes are guided in the cladding region, and there is a large difference in the radii of etched and unetched sections, the cladding modes cannot be treated as unaffected.

Numerical calculation by the finite-difference method shows that 10-40\% (depending on the wavelength and the etching radius) of energy of the first unetched fiber cladding mode propagates through the region $r_{e}<r<r_{u}$. For the second- and higher-order cladding modes, this energy increases to $50-80 \%$. As an example, the energy flux profiles of the first (solid curve) and the second (dashed curve) cladding modes of a dispersion-shifted fiber at $1300 \mathrm{~nm}$ are presented in Fig. 2(a) (normalized to unit flux over the fiber cross section). We chose $\lambda=1300 \mathrm{~nm}$ because the resonant peaks in several experiments were observed near this wavelength. ${ }^{3,11}$ The vertical dotted line shows the radius of the etched section of the fiber used in the experiments: $27 \%$ of energy flux of the first cladding mode and $74 \%$ of the second are to the right of the dotted line. Therefore, the transmission coefficients of a single unetched-etched fiber interface are $73 \%$ and $26 \%$ for the first and second cladding modes, respectively. For longer wavelengths, the mode field is farther from the core and the transmission coefficient is even smaller. It seems that propagation of cladding modes in the corrugated structure with a few tens of periods is impossible because modes lose a considerable part of their energy at each period. However, the experi- 

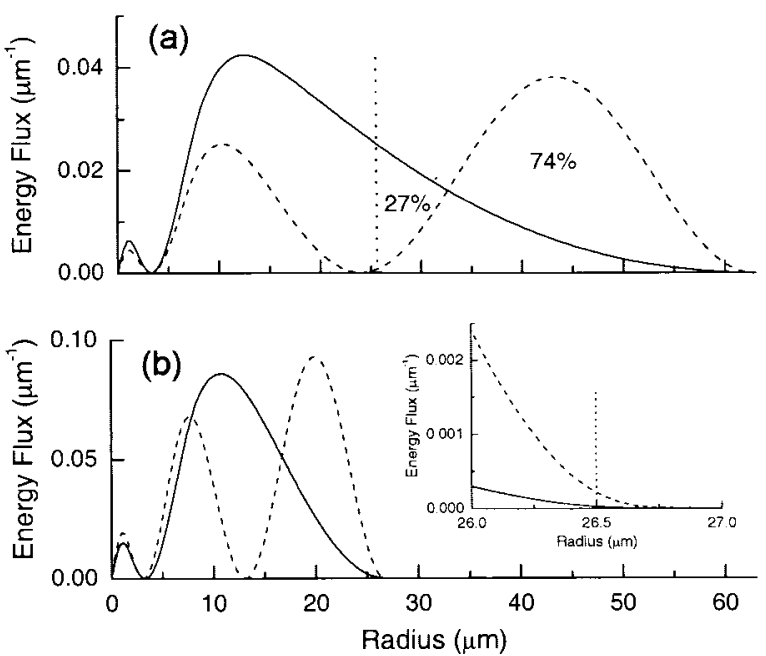

Fig. 2. Energy fluxes of the first (solid curves) and the second (dashed curves) cladding modes as functions of radius at $1300-\mathrm{nm}$ wavelength for a dispersion-shifted fiber that is (a) unetched and (b) etched with a $26.5-\mu \mathrm{m}$ radius. The energy flux over the whole fiber cross section was normalized to unity.

ments show that the cladding-mode resonance is observed at least for the first and second cladding modes, and, moreover, approximately $40 \%$ of energy coupled from the core mode into the cladding modes can be returned into the core mode for the CLPFG with more than 50 periods. ${ }^{3}$

This phenomenon can be explained as follows. The modes that propagate through the corrugated structure are in the form of eigenmodes of the etched section [Fig. 2(b)], not the unetched nor the average of etched and unetched. When these modes propagate through the unetched region they are coupled to each other and to radiation modes because they are no longer eigenmodes for this region. The overlapping integral over $r>r_{e}$ defines the coupling constants that connect the different modes. Actually a small part of mode energy propagates beyond $r_{e}$ as can be seen in Fig. 2(b). In the etched section, it is the evanescent field of the mode. Therefore, the coupling constants are rather low. Hence the field profile change is insignificant when it reaches the next etched region and effectively penetrates the etched section with small losses. At this interface, the modes are again eigenmodes and propagate without coupling.

The solution to the mode propagation problem in a twisted fiber in a linear approximation is known. ${ }^{22,23}$ The polarization of a light wave rotates during propagation through the fiber. The angle of rotation per unit length is given by

$$
\psi=-\varepsilon\left(p_{11}-p_{12}\right) \tau / 2 .
$$

This means that the eigenwaves of twisted fiber are circularly polarized waves with different propagation constants:

$$
\beta_{j \pm}{ }^{(0)}=\beta_{j}^{(0)} \pm \psi / 2 \sqrt{\varepsilon}
$$

where $\beta_{j}{ }^{(0)}$ are the propagation constants of $\mathrm{LP}_{0 j}$ modes of fiber without torsion. The electric field of circularly polarized modes can be written as

$$
\mathbf{E}_{ \pm}=\mathbf{E}_{x} \pm i \mathbf{E}_{y} .
$$

Thus, we present the electromagnetic field in the corrugated structure as a superposition of the eigenmodes of the etched section. We consider the circularly polarized waves as an unperturbed solution to the wave equation for a linearly twisted fiber and treat nonlinearity as a perturbation, which couples these circularly polarized waves.

The propagation constants of the modes in the etched section differ from those in the unetched section, ${ }^{10}$ resulting in the change of cladding-mode resonant wavelengths. In Fig. 3, the resonant wavelengths for the first and second cladding modes in the etched and unetched sections of fiber are presented for various etching radii and grating periods. The curves are obtained by numerical calculations; the points are experimental resonant wavelengths observed in fibers with and without corrugated structures. It is seen that the resonant wavelengths of the first cladding mode in the etched section shift to the longer wavelengths as compared with the ones in the unetched section. The resonant wavelengths for the second- and the higher-order modes are changed even more radically. The reason is that the energy of these modes propagates far from the core and is strongly affected by any cladding modification.

According to the coupled-mode theory, the coupling constant is given as an integral over the fiber cross section that includes the perturbation described by the change of dielectric tensor:

$$
\begin{aligned}
\kappa_{i \pm j \pm} & =\frac{k_{0}}{4} \sqrt{\frac{\varepsilon_{0}}{\mu_{0}}} \int \mathbf{E}_{i \pm} * \Delta \varepsilon \mathbf{E}_{j \pm} \mathrm{d} A, \\
\Delta \varepsilon_{k l} & =\varepsilon_{k l}^{(n l)} \text { in the etched section, }
\end{aligned}
$$

$\Delta \varepsilon_{k l}$

$$
=\left\{\begin{array}{ll}
0, & 0<r<r_{e} \\
(\varepsilon-1) \delta_{k l}, & r_{e}<r<r_{u}
\end{array}\right. \text { in the unetched section, }
$$

where the electric field is normalized to the unit flux through the fiber cross section. The cross-coupling constants between waves of right and left polarizations $\kappa_{i+j-}, \kappa_{i-j+}$ are equal to zero and $\kappa_{i+j+}=\kappa_{i-j-}$ because $\Delta \bar{\varepsilon}$ is invariable after permutation of $x$ and $y$ coordinates, i.e., it is circularly symmetric (in contrast to the linear change of the dielectric tensor induced by twist). So, only modes of the same circular polarization are coupled. Below we omit the \pm subscripts in the coupling coefficient.

For the nonperturbed fiber with only linear elasticity and linear photoelasticity, the following relation defines the resonant condition:

$$
\beta_{i \pm}{ }^{(0)}-\beta_{j \pm}{ }^{(0)}=2 \pi / \Lambda_{0},
$$




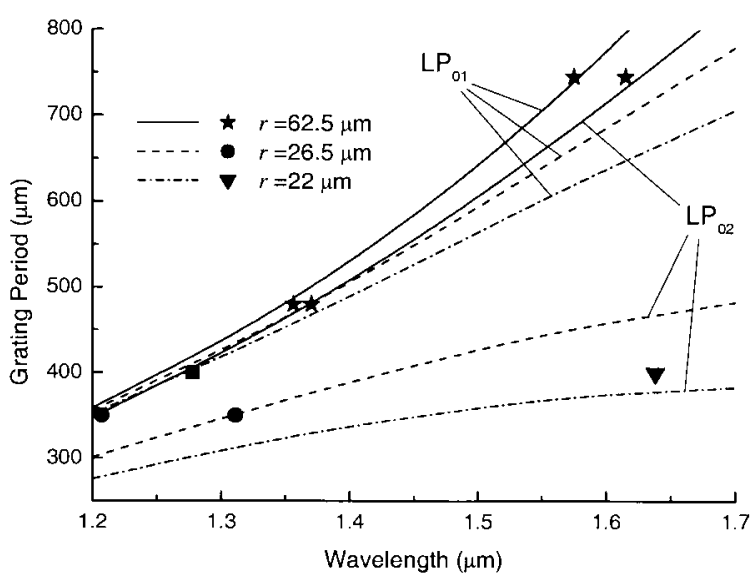

Fig. 3. Phase-matching curves for the unetched (solid curves) and the etched (dashed curves) sections of the CLPFGs for several cladding modes.

where $\Lambda_{0}$ is the nonperturbed length of the grating. Since the addition to the propagation constant $\psi / 2 \sqrt{ } \varepsilon$ in Eq. (17) is independent of the mode, the difference between the propagation constants for modes of one circular polarization is unchanged by the applied twist in the linear approximation. Therefore, the resonant condition is not changed and the wavelength is not shifted.

The resonant condition for coupling between modes of the same polarization in a grating with period $\Lambda$ can be written as

$$
\beta_{i \pm}-\beta_{j \pm}=2 \pi / \Lambda
$$

where $\beta_{i \pm}=\beta_{i \pm}{ }^{(0)}+\Delta \beta_{i} l_{e} /\left(l_{e}+l_{u}\right)$ are propagation constants presented as a sum of nonperturbed propagation constants and perturbation induced by nonlinear elasticity, nonlinear photoelasticity, and etching, which is equal to the self-coupling coefficient of the $j$ th mode:

$$
\Delta \beta_{i}=\kappa_{i i} .
$$

The addition of $\left(\Delta \beta_{i}\right)$ to the propagation constant induced by the unetched section, which is defined by an overlapping integral over the fiber cross section $r>$ $r_{e}$, is rather small $\left(\Delta n_{\text {eff }} \sim 10^{-5}\right)$ because the field outside the etched radius is evanescent and decreases exponentially. This addition does not significantly change the resonant wavelength. Besides this change is independent of the applied twist and is, therefore, constant. So, the dashed curves in Fig. 3 represent not only the resonant conditions for etched fiber but also the resonant conditions for the corrugated structure, which is confirmed by the consistency of experimental data with the calculated curves. It can be seen that the resonant wavelengths for the second cladding mode are far from the resonances of the unetched fiber. The wavelength separation between the first and the second resonances of the unetched fiber is approximately $15 \mathrm{~nm}$ and approximately $110 \mathrm{~nm}$ for the etched fiber. The measured wavelength separation of $104 \mathrm{~nm}$ proves that the modes propagate in the corrugated structure in the form of eigenmodes of the etched section.

To find the twist-dependent shift of the resonant wavelength $\Delta \lambda$ induced by nonlinear effects, we expand $\beta_{i \pm}$ in a series of $\Delta \lambda$ and use Eq. (21) to obtain

$$
\Delta \lambda=-\left(\Delta \beta_{i}-\Delta \beta_{j}\right)\left(\mathrm{d} \beta_{i} / \mathrm{d} \lambda-\mathrm{d} \beta_{j} / \mathrm{d} \lambda\right)^{-1} .
$$

Here we neglect the dependence of $\Delta \beta_{i}$ on wavelength and the change of the grating length by torsion. The difference between the gradients of core and cladding propagation constants is small, and so even a small change of dielectric tensor can produce significant shifts in resonant wavelengths.

Substituting Eq. (15) into Eq. (19), we find the self-coupling coefficient

$$
\begin{aligned}
\kappa_{i i}= & \frac{k_{0}{ }^{2}}{4 \beta_{i}} \frac{l_{e}}{l_{e}+l_{u}} \\
& \times R^{2} \tau^{2}\left[A_{r}+A_{\theta}+\left(B_{r}+B_{\theta}+\gamma_{1}+\gamma_{2}\right) I_{i}\right], \\
I_{i}= & \frac{\beta_{i}}{2 k_{0}} \sqrt{\varepsilon_{0} / \mu_{0}} \int_{0}^{r_{e}} \frac{r^{3}}{R^{2}} \mathbf{E}_{i}^{*} \mathbf{E}_{i} \mathrm{~d} r .
\end{aligned}
$$

Finally, we obtain the WS by taking into account that the difference between propagation constants of the modes is negligibly small $\left(<10^{-2}\right)$ in Eqs. (24). Then we have

$$
\begin{aligned}
\Delta \lambda & =M \tau^{2}, \\
M & =g \frac{k_{0} R^{2}\left(I_{\mathrm{co}}-I_{\mathrm{cl}}\right)}{\sqrt{\varepsilon}\left(\mathrm{d} \beta_{\mathrm{co}} / \mathrm{d} \lambda-\mathrm{d} \beta_{\mathrm{cl}} / \mathrm{d} \lambda\right)} \frac{l_{e}}{l_{e}+l_{u}}, \\
g & =\varepsilon^{2}\left(p_{11}+p_{12}\right) F-\left(\gamma_{1}+\gamma_{2}\right) / 4 .
\end{aligned}
$$

As seen from Eqs. (25), the WS is proportional to the square of the specific twist. Here we introduce parameters $g$, which defines nonlinear elasticity and photoelasticity (it does not depend on geometric parameters of fiber structure), $M$, which describes the parabola, and $I_{i}$ is the overlapping integral that depends on the field distribution of the $i$ th mode. Since the core mode is concentrated in the core region and the field is multiplied by the third power of its radius, the overlapping integral for the core mode is much smaller than that for any cladding mode.

\section{Analysis of Experimental Data}

Figure 4 shows a typical measured spectrum evolution for a CLPFG as a function of twist angle. ${ }^{13}$ The resonant wavelength shifts toward the shorter wavelength side as the twist increases. According to Eqs. (25), the wavelength shift is a parabolic function of the twist angle. Therefore, we fit the experimental data with a second-order polynomial. The fitting curve is close to most measured data. However, the center of the parabola is obviously not at zero twist. This could be due to the nonzero initial twist of a few turns in the CLPFG, which could be easily made when a 1-m-long fiber was attached to both ends. If the fiber is initially symmetric, then the WS is also 


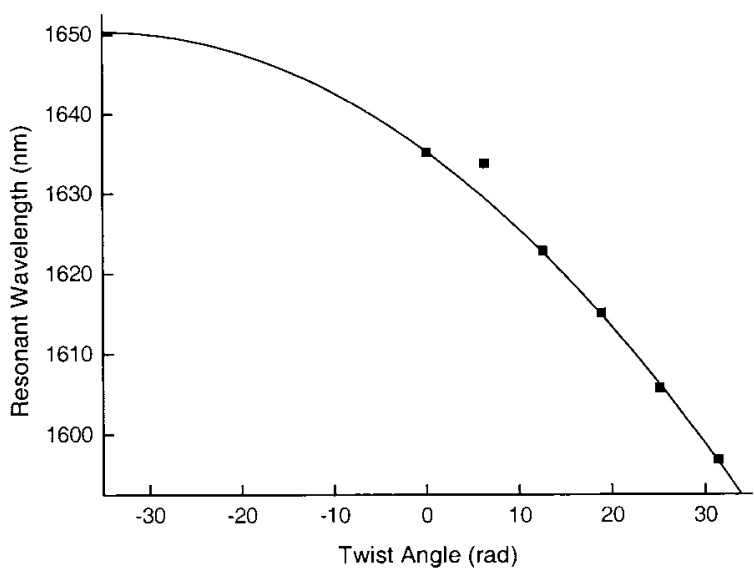

Fig. 4. Dependence of resonance WS on the applied twist rate: points, measured data; curve, best-fit parabola.

symmetric to the zero twist angle and the parabola has a vertex at the same angle.

The radii of unetched and etched sections of fiber was 62.5 and $22 \mu \mathrm{m}$, respectively. The grating was $20 \mathrm{~mm}$ long with equal lengths in etched and unetched sections. The period of CLPFG was $400 \mu \mathrm{m}$, which corresponds to the second cladding-mode resonant wavelength of approximately $1640 \mathrm{~nm}$ (see Fig. 3 ). The lengths of pristine fiber and uncoated fiber equaled 100 and $6 \mathrm{~cm}$, respectively. By suspending a torsion pendulum on bare and coated fibers of the same length, the relation between their torque was found: $k=1.10$. From these data we obtained the relation between the specific twist and the twist angle: $\tau=0.4 \phi(\mathrm{rad} / \mathrm{cm})$. When the maximum twist of $30 \mathrm{rad}$ was applied to a 1-m-long fiber, the specific twist of the etched section was $12 \mathrm{rad} / \mathrm{cm}$, which is 73 times greater than the average specific twist in unetched fiber. The parabola parameter can be found from Fig. 4: $\quad M=-0.0745 \mathrm{~nm}(\mathrm{rad} / \mathrm{cm})^{-2}$.

The linear and nonlinear elastic and linear photoelastic constants of silica have the following magnitudes ${ }^{24}$ :

$$
\begin{gathered}
\lambda=15.872 \mathrm{GPa}, \quad \mu=31.261 \mathrm{GPa} \\
l=v_{1} / 2+\nu_{2}, \quad m=\nu_{2}+2 \nu_{3}, \quad n=4 v_{3}, \\
v_{1}=54 \pm 13 \mathrm{GPa} \\
v_{2}=93 \pm 8 \mathrm{GPa} \\
v_{3}=-11 \pm 3 \mathrm{GPa} \\
p_{11}=0.113, p_{12}=0.252 .
\end{gathered}
$$

Solutions to Eqs. (4) and (5) result in the parameters that define the deformation of fiber under torsion: $F=-0.094 \pm 0.018, C=-0.279 \pm 0.015$, and $D=$ $-0.326 \pm 0.035$. From here it follows that the fiber compresses in the longitudinal direction $(D<0)$, also compresses in the transverse direction $(C<0)$, and this compression increases from the center to the edge of the fiber $(F<0)$. The compression of fiber in the longitudinal direction can be investigated by embedding a Bragg grating in the CLPFG. ${ }^{12}$
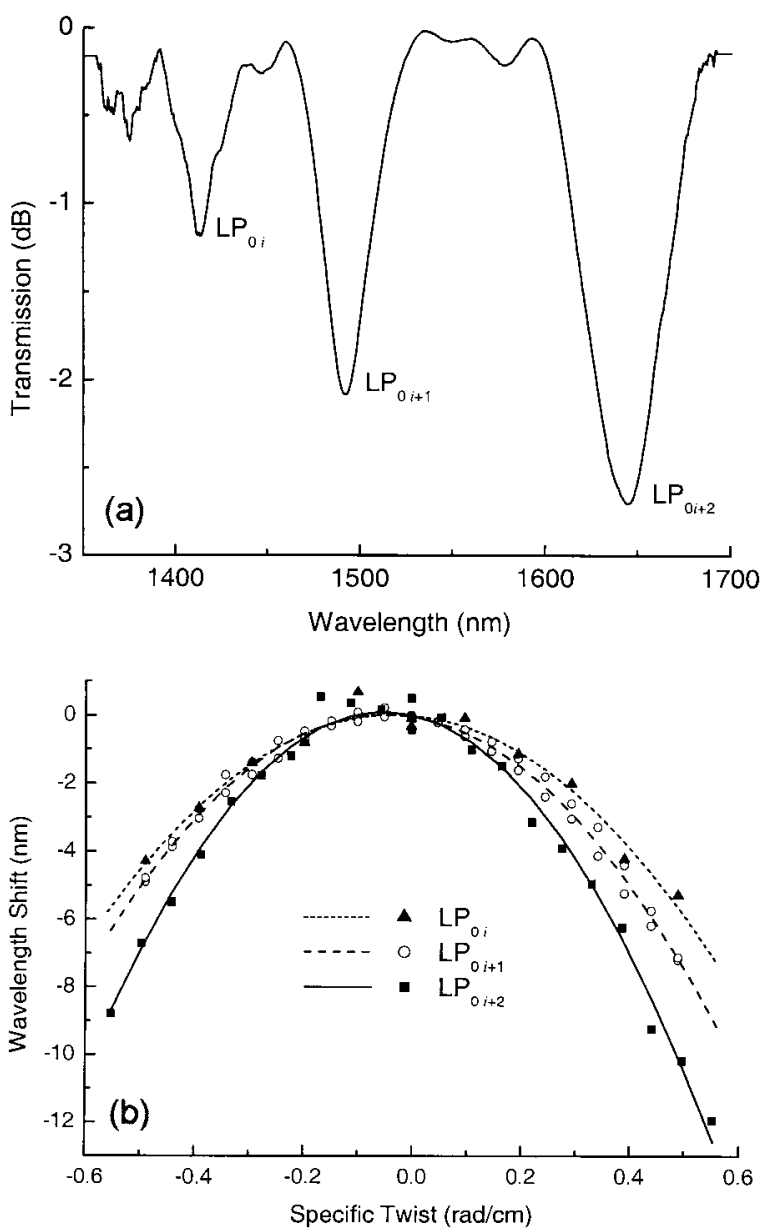

Fig. 5. (a) Typical spectrum of the PLPFG made in a photosensitive step-index fiber. (b) Dependencies of WS for three claddingmode resonances after a specific twist was applied.

The overlapping integrals for the core and first cladding modes at $1280 \mathrm{~nm}$ are $I_{\mathrm{co}}=0.028$ and $I_{\mathrm{cl}}=$ 0.382 . The experimental value of $g$ was found to be equal to $-0.20 \pm 0.04$, from which we can make an estimate of the nonlinear photoelastic constants for shearing strain: $\gamma_{1}+\gamma_{2}=0.21 \pm 0.16$.

Since the WS is the result of mode self-coupling, the same effect is also observed for a conventional photoinduced LPFG (PLPFG). For proof, we therefore investigated the spectrum behavior of a PLPFG under torsion. A PLPFG with a period of $450 \mathrm{~nm}$ and a length of $15 \mathrm{~mm}$ was made by UV radiation in a photosensitive step-index fiber. Figure 5(a) shows a typical PLPFG spectrum, where three resonant peaks can be seen. When the PLPFG was twisted, these peaks shifted. The dependence of WS on the twist angle for three resonant wavelengths is shown in Fig. 5(b). The parabolic dependencies with their branches downward are centered almost at the same the twist angle of approximately $0.5 \mathrm{rad} / \mathrm{cm}$. The dependence of the WS becomes stronger for modes of higher orders. Such behavior of the PLPFG is due mostly to lower gradients of dispersion curves for higher-order modes because the WS is inversely pro- 
Table 1. Parameters of Parabolic Functions that Describe WS and Nonlinear Photoelastic Constants for CLPFG and PLPFG

\begin{tabular}{llccc}
\hline Structure & Cladding Mode & $M, \mathrm{~nm} /(\mathrm{rad} / \mathrm{cm})^{2}$ & $g$ & $\gamma_{1}+\gamma_{2}$ \\
\hline CLPFG & $\mathrm{LP}_{\mathrm{O} 2}$ & 0.0745 & 0.2049 & 0.21 \\
PLPFG N1 & $\mathrm{LP}_{\mathrm{O}+1}$ & 0.6599 & 0.1945 & 0.17 \\
& $\mathrm{LP}_{\mathrm{O} i+2}$ & 0.9040 & 0.2230 & 0.29 \\
PLPFG N2 & $\mathrm{LP}_{\mathrm{O} i}$ & 0.5263 & 0.2220 & 0.28 \\
& $\mathrm{LP}_{\mathrm{O} i+1}$ & 0.6270 & 0.1754 & 0.09 \\
& $\mathrm{LP}_{\mathrm{O} i+2}$ & 0.8791 & 0.1938 & 0.17 \\
\hline
\end{tabular}

portional to this slope [see Eq. (23)]. We also observed that the WS is approximately proportional to the peak width.

Equation (24) can also be applied for the PLPFG with $l_{u}=0$. As can be seen from Eqs. (25), the parameter of nonlinearity $g$ does not depend on the geometric parameters of fiber but only on elastic and photoelastic constants of fiber glass. This parameter can be found from the measured WS. It has to be the same for different kinds of gratings and for various cladding-mode resonances. The results of processed data for both CLPFG and PLPFG are summarized in Table 1 . The magnitudes of parameter $g$ are quite close to each other. Taking into account data of all the resonances, we found that $g=$ $-0.202 \pm 0.018$ and $\gamma_{1}+\gamma_{2}=0.20 \pm 0.07$. To our knowledge this is the first estimation of nonlinear photoelastic parameters of silica for shear strain.

\section{Conclusion}

We have investigated the wavelength shift of cladding-mode resonance in corrugated long-period fiber gratings under torsion. The wavelength shift is explained as the result of nonlinear elastic and quadratic photoelastic effects. The nonlinear elastic behavior of fiber under torsion is analyzed based on the Murnaghan model of a solid elastic body. The quadratic photoelastic effect described by the displacement gradient variable was used to determine the permittivity tensor of a twisted fiber and compared with the classical quadratic photoelastic effect described by the strain variable. We show that the classical tensor of quadratic photoelasticity is symmetrized and includes an additional term that is proportional to the first-order photoelastic tensor. The quadratic tensor of photoelasticity in displacement gradient representation has seven independent constants for isotropic media. The electromagnetic field in the corrugated structure is presented as a superposition of circularly polarized modes of the etched section of the fiber. The self-coupling of these modes induced by nonlinear effects changes the phasematching condition so that the cladding-mode resonances shift to shorter wavelengths. The amount of wavelength shift determined by nonlinear elasticity and quadratic photoelasticity constants is proportional to the square of the twist angle, which is in agreement with experimental results. Based on our theory, we have predicted and proved experimentally the existence of the wavelength shift for a conven- tionally photoinduced LPFG. For the first time to our knowledge, quadratic photoelastic constants of fiber glass for shearing strain have been found from both corrugated and photoinduced LPFGs. Within the limits of experimental errors, these constants are consistent in value, which strongly supports the underlying mechanisms we have explained.

The authors are grateful for the support by the National Science Council Taiwan, under project NSC 91-2215-E-002-028 and by the Education Ministry of Taiwan under project EDU 89-E-FA06-2-4-7.

\section{References}

1. A. M. Vengsarkar, P. J. Lemaire, J. B. Judkins, V. Bhatia, T. Erdogan, and J. E. Sipe, "Long-period fiber gratings as bandrejection filters,” J. Lightwave Technol. 14, 58-65 (1996).

2. V. Bhatia and A. M. Vengsarkar, "Optical fiber long-period grating sensors," Opt. Lett. 21, 692-694 (1996).

3. C. Y. Lin, L. A. Wang, and G. W. Chern, "Corrugated longperiod fiber gratings as strain, torsion, and bending sensors," J. Lightwave Technol. 19, 1159-1168 (2001).

4. A. M. Vengsarkar, J. R. Pedrazzani, J. B. Judkins, P. J. Lemaire, N. S. Bergano, and C. R. Davidson, "Long-period fibergrating-based gain equalizers," Opt. Lett. 21, 336-338 (1996).

5. C. E. Chryssou, "Gain-equalizing filters for wavelength division multiplexing optical communication systems: a comparison of notch and long-period grating filters for integrated optoelectronics," Opt. Commun. 184, 375-384 (2000).

6. G. W. Chern, L. A. Wang, and C. Y. Lin, "Transfer-matrix approach based on modal analysis for modeling corrugated long-period fiber gratings," Appl. Opt. 40, 4476-4486 (2001).

7. Y. Liu, L. Zhang, and I. Bennion, "Fibre optic load sensors with high transverse strain sensitivity based on long-period gratings in B/Ge co-doped fibre," Electron. Lett. 35, 661-663 (1999).

8. Y. Liu, L. Zhang, J. A. R. Williams, and I. Bennion, "Optical bend sensor based on measurement of resonance mode splitting of long-period fiber grating," IEEE Photon. Technol. Lett. 12, 531-533 (2000).

9. B. H. Lee, Y. Liu, S. B. Lee, S. S. Choi, and J. N. Jang, "Displacements of the resonant peaks of a long-period fiber grating induced by a change of ambient refractive index," Opt. Lett. 22, 1769-1771 (1997).

10. S. Kim, Y. Jeong, S. Kim, J. Kwon, N. Park, and B. Lee, "Control of the characteristics of a long-period grating by cladding etching," Appl. Opt. 39, 2038-2042 (2000).

11. L. A. Wang, C. Y. Lin, and G. W. Chern, "A torsion sensor made of a corrugated long period fibre grating," Meas. Sci. Technol. 12, 793-799 (2001).

12. C. Y. Lin, G. W. Chern, and L. A. Wang, "Periodical corrugated structure for forming sampled fiber Bragg grating and longperiod fiber grating with tunable coupling strength," J. Lightwave Technol. 19, 1212-1220 (2001).

13. C. Y. Lin and Lon A. Wang, "A wavelength- and loss-tunable band-rejection filter based on corrugated long-period fiber grating," IEEE Photon. Technol. Lett. 13, 332-334 (2001).

14. C. Y. Lin, G. W. Chern, and L. A. Wang, "Periodical corrugated structure for forming sampled fiber Bragg grating and longperiod fiber grating with tunable coupling strength," J. Lightwave Technol. 19, 1212-1220 (2001).

15. R. J. Atkin and N. Fox, An Introduction to the Theory of Elasticity (Longmans Green, New York, 1980).

16. F. D. Murnaghan, Finite Deformation of an Elastic Solid (Wiley, New York, 1951).

17. T. S. Narasimhamurty, Photoelastic and Electro-Optic Properties of Crystals (Plenum, New York, 1981). 
18. D. F. Nelson and M. Lax, "Theory of photoelastic interaction," Phys. Rev. B 3, 2778-2794 (1971).

19. K. Vedam and R. Srinivasan, "Non-linear piezo-optics," Acta Crystallogr. 22, 630-634 (1967).

20. G. N. Burlak, V. V. Grimal'skii, and K. Ishkabulov, "Dynamics of acoustoelectromagnetic solitons as a manifestation of higher nonlinearity," Phys. Solid State 39, 987-990 (1997).

21. K. Vedam, E. D. D. Schmidt, and R. Roy, "Nonlinear variation of refractive index of vitreous silica with pressure to 7 Kbars," J. Am. Ceram. Soc. 49, 531-535 (1971).

22. A. M. Smith, "Birefringence induced by bends and twists in single-mode optical fiber," Appl. Opt. 19, 2606-2611 (1980).

23. R. Ulrich and A. Simon, "Polarization optics of twisted singlemode fibers," Appl. Opt. 18, 2241-2251 (1979).

24. A. I. Lurie, Nonlinear Theory of Elasticity (Elsevier, Amsterdam, 1990). 